\title{
Identifying Critical Success Factors for the Implementation of a Social Knowledge Management in the Public Sector
}

\author{
Timo Hammerl \\ University of Regensburg \\ Timo.hammerl@ur.de
}

\begin{abstract}
Since knowledge is considered to be one of the most important resources of an organization, the need for successful management of this resource has become crucial. In the current era of social media, new possibilities exist for enhancing knowledge management (KM) via collaboration and interaction facilitation. With a model for successfully implementing a social KM system at hand, the author matches critical success factors from the areas of $K M$, enterprise social networks, and online social networks to technological, organizational, social and individual dimensions which form the so-called TOSI framework via the design science research approach. Resulting in the adaption of a framework for a KM context. The applicability of the adapted framework is demonstrated by using it for the implementation process of a wiki at a public sector organization.
\end{abstract}

\section{Introduction}

In the $21^{\text {st }}$ century, the necessity of getting the right knowledge at the right time for the fulfillment of a task or job is crucial. Similarly, with the advent of social media and worldwide networking the world has transformed into a hyper-speed environment where societies and organizations are able to retrieve information whenever it is needed. Therefore, providing great products is not the only focus of organizations anymore, but rather the provision of knowledge and information without any delays for the respective customers or audience (e.g., customer service). If this provision is not complied with, consequences such as potential customer or reputation loss become conceivable. In this regard, knowledge can be considered to be one of the most important resources of an organization to this day [1]. By looking at both private and public organizations, the importance of this resource becomes even more vivid. [2] analyze the improved knowledge-sharing and problem-solving abilities of professional service firms (PSFs) through the implementation of knowledge facilitating tools such as an enterprise social network (ESN). They state that "the key to success of PSFs is reliant on the organizational knowledge-sharing practices." They further argue the importance of attracting, mobilizing, developing and transforming employees' knowledge for the delivery of valuable client-focused services. Additionally, public sector organizations are widely regarded as knowledge-based organizations that focus on developing and providing knowledge for stakeholders [3]. This conforms with the statement from [1], where knowledge is considered to be a key resource. For processes and operations of public sector organizations to run in the most effective manner, and to hold bureaucratic efforts to a minimum, it is essential to minimize processing times via the proper management of its most important resource. Additionally, since the average working age in public sector organizations tends to be very high, the risk of knowledge loss due to retirement rises exponentially without proper knowledge management (KM). Therefore, adequate KM with state-of-the-art IT technology and integrated processes that ensure effective knowledge exchange and knowledge conservation is essential.

Admittedly, KM no longer a new research field [cf. 4]. However, as [1] stated, a classical knowledge management system (KMS) often lacks social components, such as interaction possibilities. Therefore, the use of social media technologies for $\mathrm{KM}$ has resulted in a sort of renaissance for this research area [5] since it helps with facilitating collaboration resulting from simplified interaction functionalities. Nevertheless, the implementation of an organization-wide KMS can be cost-intensive and time-consuming if it does not have a proper strategy or model to draw upon. In this matter success factors assist by providing suggestions on what to consider in order for the implementation to go smoothly. To the best of the author's knowledge, there is no holistic framework that considers all possible success factors for a social KMS implementation. This gap is closed by providing a comprehensive overview of 
relevant critical success factors (CSFs), where the technological, organizational, social and individual (TOSI) framework sets the scene for a holistic approach, which can be used as guidance for implementing a social KMS.

Furthermore, the author accompanied the implementation process of KM at a German public sector organization and applied the framework from this paper in order to demonstrate its applicability in practical settings. Furthermore, [6] state that there is an overabundance of publications from Asia dealing with $\mathrm{KM}$ in the public sector, making the need for another demonstration location essential. Additionally, this German public sector organization initiated its own approach for introducing a KMS (more specifically a wiki) to the organization in the past, but did not have a clear scope or strategy (provide-and-pray approach [7]). Because of this, additional insights into challenges and potential solutions for a promising revision of a KM implementation with the use of success factors were gained.

Considering the described research gaps above, the following research questions were formulated:

RQ1: Which CSFs exist in the context of a social KMS and how can they be classified?

RQ2: Which CSFs are considered relevant for a social KMS implementation at a public sector organization?

The structure of the paper is as follows: Section 2 provides an understanding of the conceptional basics for the context of this paper. Related work is covered in Section 3 by considering research on wikis and the public sector. In Section 4, the procedure of the research is presented. The adapted framework is presented in Section 5 and is demonstrated on a German public sector organization in Section 6 . Limitations as well as an outlook are discussed in section 7.

\section{Conceptional Basics}

In research, a broad range of definitions for the term knowledge management is available. [8] devoted a whole chapter to the definition of $\mathrm{KM}$ in his book. According to him, KM is defined "....as the practice of selectively applying knowledge from previous experiences of decision making to current and future decision-making activities with the express purpose of improving the organization's effectiveness". He further describes the term KMS as a "system created to facilitate the capture, storage, retrieval, and reuse of knowledge." Within the era of social media, KM is complemented by social media platforms that support sharing, co-creation and discussion, which are key knowledge processes [9]. Therefore, the combination of social media and $\mathrm{KM}$ is referred to as social $\mathrm{KM}$ [10].

In terms of $\mathrm{KM}$, collaboration technologies play an essential role for the successful distribution and use of knowledge. [11] already classified wikis as being a form of social media technology. More specifically, the wiki technology exemplifies the dynamic and collaborative characteristics of Web 2.0, which extends the capability of internet-based computing to improve communication and collaboration across time and distance by maintaining user updates relating to available articles [12].

In order to determine success, a well-established approach is the use of CSFs. Therefore, [13] state that "CSFs are the limited number of areas in which satisfactory results will ensure successful competitive performance for the individual, department or organization. CSFs are the few key areas where 'things must go right' for the business to flourish and for the manager's goals to be attained."

The basis of the social KMS implementation forms the TOSI dimensional framework, initial presented by [2]. According to [2], the original idea of this framework was based upon the research of [14] and [15], both of whom used innovation diffusion theory and social capital theory as starting points for their developments. As described by [15], three dimensions (technological, organizational and social) could be retrieved. Further, through the case study analysis method, [14] also identified the individual dimension as being essential. The basis of their development was the IS impact measurement model of [16], who suggested four success dimensions for measuring system success: individual impact, organizational impact, system quality and information quality.

Regarding the TOSI framework, the technological dimension summarizes all factors that relate to some sort of physical or technical characteristic of the KM solution. However, it must be noted that KM per se is far more than simply technical. In fact, some authors even consider IT and by extension the technical dimension as being the least important since people and processes are seen as key to successful KM [17] [5]. Nonetheless, it is undeniable that technological factors build the foundation of a working KMS.

The organizational dimension contains all of the factors concerning organizational processes and the environment that impact the use of the KMSs, according to [2]. The root of this dimension's definition goes back to the initial research regarding 
social capital theory, where it was defined as contextual factors [cf. 15, 18]. However, this view is extended by considering not only factors such as organizational climate but also factors regarding the implementation of processes and strategies.

The social capital theory provides the basis for the social dimension. According to [19], social capital represents a set of social resources, such as norms, trust and values, embedded in relationships to benefit individuals' actions. Additionally, as already state in 2001 by [20], social capital has been shown to be positively associated with knowledge acquisition. Therefore, the social dimension comprises all factors relating to social resources.

Apart from social capital theory, the individual dimension as defined by [14] is also critical for a successful KM implementation. This is justified by referring to the widely established IS success model by [21] and its extension by [16]. The authors introduced relevant dimensions for the measurement of IS success, where the concept from [14] is relied upon. The individual dimension contains factors such as individual motives or the IT skills of employees.

\section{Related Work}

As already noted, collaboration technologies such as wikis have developed a certain popularity in the context of KM. Therefore, several authors have examined the use and acceptance of wikis in organizations. However, most often these researchers only focused on specific aspects for a successful wiki implementation, such as the technical aspects that have been considered by diverse authors [e.g., 12, 22]. This seems paradox given that wikis are generally considered to be simple, intuitive and easy to use [e.g., 23]. Furthermore, [12] provides an extension to the theory of innovation diffusion by arguing users' acceptance of a wiki system in the context of KM. He does so by extending the technology acceptance model [24] with social factors. As a result, this approach has led to the implicit consideration of two dimensions of the TOSI framework: technological and social. Likewise, [25] addresses social factors in the context of the acceptance and use of Web 2.0 tools for collaboration. However, they solely focus on social mechanisms, which results in the neglection of other relevant dimensions. More concretely, the authors identify the critical mass as being the strongest factor for the acceptance of Web 2.0 tools and, therefore, propose a more detailed analysis of it. Other researchers, such as [22], have focused on the use and adaption of a wiki for small and medium sized enterprises (SMEs) and validated its usefulness in practical settings. Their approach uses explicitly identified wiki CSFs that consider the key obstacles for a successful implementation as defined by [26] (e.g., low usability, lack of clear purpose and others). [27] investigates the adoption of an enterprise wiki for knowledge creation and diffusion purposes using a holistic approach that considers the influence of technological, personal and organizational factors. As one result, they state that out of five potential factors, only three are considered relevant: top management support, technical support and knowledge sharing self-efficacy. Even when taking the above-mentioned authors into account, there still remains the lack of a holistic framework that considers all aspects (dimensions) of the implementation of a social KMS (e.g. a wiki) in research.

In addition to considering the implementation of a wiki for a social KMS with respect to the adapted TOSI framework, the approach is also demonstrated on a public sector organization. In this respect, [6] conducted an extensive literature review regarding $\mathrm{KM}$ in the public sector. The authors' intention is twofold; they want to inform practitioners and academics about the main evolution in this field and highlight future research needs. Among their findings is the fact that public sector $\mathrm{KM}$ literature is fragmented and dominated by unrelated research, with a distinct focus on Malaysian and Indian organizations. According to [6], these countries are populous but hardly leaders in the field.

Furthermore, [5] state that only $0.33 \%$ of KM research regarding the public sector provides practical insights, leaving a huge gap between theory and practice. One up-to-date example of KM in the public sector is described in [28]. They examined the effect of knowledge-sharing propensity on knowledge-sharing behavior and individual work performance among employees in a public sector organization. However, they solely focused on knowledge distribution, resulting in the neglection of essential components, such as the identification, development or storage of knowledge.

\section{Methodology}

For the adaption of the TOSI framework and its test in practical settings, the design science research (DSR) approach by [29] was followed.

In phase one, problems were identified by unveiling the need for a holistic CSF approach for a social KMS implementation. Phase two defined the objective of the solution, which is the adaption of the TOSI model to generate a holistic framework for 
social KMS implementation and its application to a public sector organization.

To design and develop (phase 3 ) the solution, the author first consolidated different existing CSF literature reviews in the areas of KM, ESN and OSN. More specifically, [30, 31] provided two extensive overviews in the field of KM and CSFs. However, since the importance of social media functionalities in a KM context needs to be stressed, the scope of the literature search was extended to include CSFs regarding an ESN context as well as an OSN context. Fortunately, the author could rely on the literature reviews by $[2,15,32,33]$ for ESN context. To address OSN CSFs, the literature review by [34], who identified and categorized CSFs in an OSN context, was referred to. After analyzing and consolidating the literature, the CSF results were matched to according dimensions of the TOSI framework. It is important to mention that, up to this point, there were still many duplicates because of the close relationship between ESN and OSN, as well as their interdependency to KM. Therefore, similar CSFs were grouped and duplicates were eliminated according to the qualitative content analysis by [35] (see Section 5 for details).

For the demonstration and evaluation (phases four and five) of the framework adaption, the author accompanied the implementation of a social KMS at a German public sector organization, which provided an opportunity to assess the process as well as get insights into the applicability of the framework in practical settings.

The publication of the results (phase six: communication) is also part of this article.

\section{Findings and Results}

As for the KM context, two extensive publications that explored and combined literature on CSFs of KM were drawn upon. In 2005, [31] conducted pioneering research in the field of $\mathrm{KM}$ CSF research by considering all possible CSFs up to 2004. As a result, the authors combined similar CSFs into composite CSFs, which resulted in a total of 12 CSFs for further consideration. These factors were then ranked based on the number of authors mentioning the factors. As an addition to this pioneering research, [30] extended the timeframe to include relevant literature from 2004 to 2016 based on a qualitative research approach. This resulted a total of 702 CSFs from the analysis of 72 research papers for further clustering. After they grouped similar CSFs together, a total of 24 CSFs remained. Notably, however, the current author specifically refers to the penultimate step of [30], since their final proposal of nine KM CSFs would have been too generic for the context of this paper. After comparing and consolidating the results of [31] and [30], the following KM CSFs could be retrieved (see Table 1).

Table 1. KM CSFS

\begin{tabular}{ll} 
ID & KM CSFs \\
\hline KM01 & Providing state-of-the-art IT [30] [31] \\
\hline KM02 & KM culture [30] [31] \\
\hline KM03 & KM strategy [30] [31] \\
\hline KM04 & Leadership [30] [31] \\
\hline KM05 & KM process [30] [31] \\
\hline KM06 & KM organization [30] \\
\hline KM07 & Motivation [30] [31] \\
\hline KM08 & HR Management [30] \\
\hline KM09 & Training [30] [31] \\
\hline KM10 & KM resources [30] \\
\hline KM11 & KM measurement [30] [31] \\
\hline KM12 & Communication [30] \\
\hline KM13 & KM structure [30] [31] \\
\hline KM14 & Incentives [30] [31] \\
\hline KM15 & Employee involvement [30] \\
\hline KM16 & Teamwork [30] \\
\hline KM17 & Benchmarking [30] \\
\hline KM18 & Trust [30] \\
\hline KM19 & Learning organization [30] [31] \\
\hline KM20 & KM expert(s) [30] \\
\hline KM21 & Clear goal and purpose [31] \\
\hline KM22 & Security + protection of knowledge [31] \\
\hline KM23 & Easy knowledge use through good usability [31] \\
\hline
\end{tabular}

As shown, [31] have already identified the majority of CSFs in KM context with an additional three CSFs (KM21-KM23) that were not listed by [30]. Inversely, [30] identified CSFs (e.g., KM06, KM08, KM10, KM12, KM15, KM16-18, KM20) that were not mentioned by [31]. However, it needs to be emphasized that the relevance or completeness of these two literature reviews has not been assessed, and neither should it be assumed that one review is better than the other. Rather, both authors share a large consensus relating to KM CSFs.

Regarding CSFs in the context of an ESN, an initial literature review was conducted by [15], who had already referred to the usage of three TOSI framework dimensions (technological, organizational and social) and had identified nine possible CSFs. However, when considering literature after 2012, three additional publications were found. In 2015, [2] explored factors influencing the usage of an ESN in PSFs. In this regard, the authors matched the identified factors to the TOSI dimensions. They aggregated their findings in order to form a 
comprised overview. However, due to this aggregation, essential information for the matching process (described later) have been lost. Therefore, the non-aggregated list of [2] is referred to. In this matter, they differentiated between enabler and inhibitor factors. In order to get the most extensive view for this paper, the mentioned inhibitors were inverted to retrieve some additional CSFs for the context of an ESN. This approach resulted in a total of $25 \mathrm{CSF}$ to be considered. Similarly, [32] examined what influences employees to use ESNs. Since papers [2] and [32] where written by the same authors, the majority of ESN CSFs could be adopted. However, [32] provided two additional factors to be considered. These are education and training and content quality (the latter could be grouped with information quality since it roughly means the same (see ESN18)). Lastly, [33] matched the existing CSFs of an ERP implementation process to the ESN context, resulting in a total of 11 CSFs. However, these identified factors are very generic and are applicable to any software implementation project. Therefore, only some CSFs are considered relevant for the paper, such as teamwork, top management. support, performance measurement and project management. The four publications were consolidated in order to group similar CSFs and to eliminate duplicates. The final list of ESN CSFs is shown in Table 2.

\begin{tabular}{ll} 
Table 2. ESN CSFs & $\begin{array}{l}\text { ESN CSFs } \\
\text { ID }\end{array}$ \\
\hline ESN01 & Relative advantage [15] \\
\hline ESN02 & Result demonstrability [15] \\
\hline ESN03 & Compatibility [15] \\
\hline ESN04 & Trust [15] \\
\hline ESN05 & Community identification [15] \\
\hline ESN06 & System integration [2, 32] \\
\hline ESN07 & Accessibility [2, 32] \\
\hline ESN08 & Functionality scope [2, 32] \\
\hline ESN09 & Ease of use (Usability) [2, 15, 32] \\
\hline ESN10 & Perceived security [2, 32] \\
\hline ESN11 & Top management support [2, 33] \\
\hline ESN12 & ESN strategy [2] \\
\hline ESN13 & Reward system [2, 32] \\
\hline ESN14 & Policies [2] \\
\hline ESN15 & Awareness campaign [2, 32] \\
\hline ESN16 & Critical mass [2, 15, 32] \\
\hline ESN17 & Reciprocity [2, 32] \\
\hline ESN18 & Information + content quality [2, 32] \\
\hline ESN19 & Task characteristics [2, 32] \\
\hline ESN20 & Tolerance of failure [2, 32] \\
\hline ESN21 & Supportive community member [2, 32] \\
\hline ESN22 & Collaborative norms [2, 15, 32] \\
\hline ESN23 & Sense of connectness [2, 32] \\
\hline EN
\end{tabular}

\begin{tabular}{ll}
\hline ESN24 & Social ties $[2,32]$ \\
\hline ESN25 & Reputation $[2,15,32]$ \\
\hline ESN26 & Enjoy. helping others $[2,32]$ \\
\hline ESN27 & Personality $[2,32]$ \\
\hline ESN28 & Time $[2,32]$ \\
\hline ESN29 & ESN skills $[2,32]$ \\
\hline ESN30 & Knowledge self-eff. $[2,32]$ \\
\hline ESN31 & Education and training [32] \\
\hline ESN32 & Performance measurement [33] \\
\hline ESN33 & Project management [33] \\
\hline ESN34 & Teamwork [33]
\end{tabular}

To get the most extensive view on social KMS CSFs, the author found it promising to analyze CSFs of OSNs. Therefore, the work of [34], who conducted an extensive literature review on CSFs in the context of OSNs, was drawn upon. However, the scope of this publication is limited to the management of internal knowledge that has resulted in the neglect of CSFs regarding the customer's (B2C) perspective. Therefore, out of 42 CSFs, 22 were considered not relevant. For example, the CSFs unprofessionalism and cheap advertisement have the right of existence in the $\mathrm{B} 2 \mathrm{C}$ context, where one aim is to reach and engage with as many potential users as possible. However, in the context of internal KM, these CSFs do not matter much. As another example, providing up-to-date content (e.g., breaking news posts) does not play a significant role in $\mathrm{KM}$ since $\mathrm{KM}$ is not solely about providing the newest information but more importantly about the providing necessary information (which could also be older). Altogether, a total of 20 relevant OSN CSFs could be retrieved. Table 3 provides an overview.

As an initial step for the adaption of the TOSI framework, duplicates were eliminated, as the three lists contained many redundancies (e.g., top management support was considered in all three categories). For further reduction, the qualitative content analysis by [35] was drawn upon to find and develop adequate groups. For example, KM12, KM15, ESN05, ESN17, ESN22, ESN24, OSN15, OSN18, OSN19 were grouped together and renamed interactivity and collaboration since each single CSF of the three categories means roughly the same thing. Another example is the summarization of the ESN18 and OSN20 CSFs to quality content. The steps of duplicate elimination and grouping resulted in a total of $38 \mathrm{CSFs}$ for the TOSI framework matching process.

To reduce subjectivity, the author consulted two other researchers in order to discuss the reduction and matching approach and, afterwards, to reach a consensus. 
As such, one discrepancy lay in the way of matching the CSF information + content quality to an adequate dimension. One researcher suggested to match the CSF to the individual dimension, whereas the other researcher found it more suitable to match it to the social dimension. However, after some discussion, the consensus was to allocate this CSF to the social dimension since the researchers agreed that qualitative content and information will eventually result in the readiness to contribute and interact with other users.

Table 3. OSN CSFs

\begin{tabular}{ll}
\hline ID & OSN CSFs \\
\hline OSN01 & Privacy protection [34] \\
\hline OSN02 & Personalization [34] \\
\hline OSN03 & Providing no alternatives [34] \\
\hline OSN04 & Management support [34] \\
\hline OSN05 & Set up guidelines / Netiquette [34] \\
\hline OSN06 & Conduct workshops [34] \\
\hline OSN07 & Identify and determine KPIs [34] \\
\hline OSN08 & HR for planning and implementation [34] \\
\hline OSN09 & Define responsibilities [34] \\
\hline OSN10 & Committed team [34] \\
\hline OSN11 & Establish project management [34] \\
\hline OSN12 & Building a reputation [34] \\
\hline OSN13 & (Web) Application knowledge [34] \\
\hline OSN14 & Benefit for the individual [34] \\
\hline OSN15 & Be active [34] \\
\hline OSN16 & User-friendliness [34] \\
\hline OSN17 & Cultural consideration [34] \\
\hline OSN18 & Collaboration [34] \\
\hline OSN19 & Interactivity [34] \\
\hline OSN20 & Provide qualitative content [34] \\
\hline
\end{tabular}

Due to page restrictions, the detailed matching process cannot be described and demonstrated in further detail. However, to get a basic understanding of the underlying thoughts of the matching procedure, one CSF per dimension is explained below.

The allocation of the CSF usability to the technological dimension can be substantiated with the fact that an IT product does not only have to fulfill functional requirements, but also needs to be designed in a way that is appealing to the users. Therefore, the newest design and IT standards should be considered in order to ensure user acceptance. Furthermore, establishing an adequate organizational culture and structure could be matched to the organizational dimension. This means that for KM to function, diverse general conditions in the culture of an organization need to be fulfilled. For example, the importance of KM needs to be embedded in the organization as well as underlying reporting structures or the recruitment of a knowledge manager needs to be considered. As far as the social dimension is concerned, the CSF critical mass could be matched to this dimension. As long as there are not enough people contributing or there is not enough content available, both problems will prevent other people from contributing as well. Meaning that after a critical mass is reached, the collaboration aspect will significantly increase as well since the wait-andsee behavior will vanish. Finally, the matching of the CSF individual motives to the individual dimension is justified with the name of the dimension itself. For, in general, every person has different reasons why they want to contribute. Sometimes it is about building a reputation or gain recognition, while in other cases people just enjoy helping others. The final TOSI framework with all matched CSFs is shown in Table 4.

\section{Demonstration in Practice}

Being a service provider, the family benefits department of the Federal Employment Agency of Germany relies heavily on the provision of information and the distribution of knowledge. However, until now, no specific KM activities have been implemented in this organization. With this in mind, the family benefits department (responsible for providing child benefits to parents with approx. 3600 employees) launched a project in collaboration with the author (in the role of KM expert and consultant) and a university to implement a nationwide KMS for the capturing, storing, retrieving, reusing and sharing of knowledge. Furthermore, in an initial analysis, the department identified that up to $30 \%$ of its current workforce will be retiring in the next years. Which, in other words may result in a tremendous amount of knowledge loss if this resource is not managed properly. Also, the organization's vision is to become the best service provider of social benefits in Europe. In order to achieve this goal, it is necessary to shift its focus from being a mass administration toward the development of a quality administration to which KM is essential.

Being an organization of the public sector, an additional challenge regarding the technical perspective had to be dealt with. Due to data privacy concerns, the organization prohibited the implementation of cloud technologies of any kind.

This matter needs to be emphasized since it played a major role in the decision of what KMS to implement and develop. Therefore, an on-premise implementation of an organization-wide wiki seemed most promising for the success of the project. 
Table 4. TOSI Framework

\begin{tabular}{|c|c|c|c|c|}
\hline \multicolumn{2}{|c|}{ Technical } & \multicolumn{2}{|l|}{ Organizational } & Individual \\
\hline \multirow{14}{*}{ 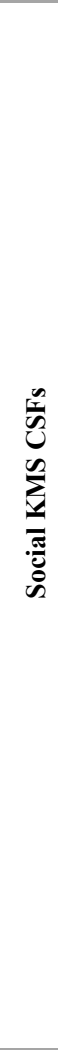 } & $\begin{array}{l}\text { Providing State-of-the-Art IT } \\
\text { (KM01) }\end{array}$ & $\begin{array}{l}\text { Top Management. Support } \\
\text { (KM04, ESN11, OSN04) }\end{array}$ & $\begin{array}{l}\text { Critical Mass } \\
\text { (ESN16) }\end{array}$ & $\begin{array}{l}\text { Individual Motives } \\
\text { (ESN25, ESN26, OSN12) }\end{array}$ \\
\hline & System Integration (ESN06) & $\begin{array}{l}\text { Develop a Strategy + Goal } \\
\text { (KM03, KM12, ESN12, ESN33) }\end{array}$ & $\begin{array}{l}\text { Teamwork Culture } \\
\text { (KM16, ESN34, OSN10) }\end{array}$ & $\begin{array}{l}\text { Individual Characteristics } \\
\text { (KM07, ESN27) }\end{array}$ \\
\hline & $\begin{array}{l}\text { Accessibility } \\
\text { (ESN07) }\end{array}$ & $\begin{array}{l}\text { Reward System } \\
\text { (KM14, ESN13) }\end{array}$ & $\begin{array}{l}\text { Tolerance of Failure } \\
\text { (ESN20) }\end{array}$ & $\begin{array}{l}\text { Time } \\
\text { (ESN28) }\end{array}$ \\
\hline & $\begin{array}{l}\text { Usability } \\
\text { (KM23, ESN09, OSN16) }\end{array}$ & $\begin{array}{l}\text { Awareness Campaign } \\
\text { (ESN15) }\end{array}$ & $\begin{array}{l}\text { Supportive Community Member } \\
\text { (ESN21) }\end{array}$ & $\begin{array}{l}\text { IT Skills } \\
\text { (ESN29, OSN13) }\end{array}$ \\
\hline & $\begin{array}{l}\text { Security } \\
\text { (KM22, ESN10, OSN01) }\end{array}$ & $\begin{array}{l}\text { Policies and Guidelines } \\
\text { (ESN14, OSN05) }\end{array}$ & $\begin{array}{l}\text { Sense of Connectness } \\
\text { (ESN23) }\end{array}$ & $\begin{array}{l}\text { Knowledge } \\
\text { (KM20, ESN30) }\end{array}$ \\
\hline & $\begin{array}{l}\text { Personalization } \\
\text { (OSN02) }\end{array}$ & $\begin{array}{l}\text { Education and Training } \\
\text { (KM09, ESN31, OSN06) }\end{array}$ & $\begin{array}{l}\text { Interactivity and Collaboration } \\
\text { (KM12, KM15, ESN05, ESN17, } \\
\text { ESN22, ESN24, OSN15, OSN18, } \\
\text { OSN19) }\end{array}$ & $\begin{array}{l}\text { Advantage for Work } \\
\text { (ESN01, OSN14) }\end{array}$ \\
\hline & Functionality Scope (ESN08) & $\begin{array}{l}\text { Trust } \\
\text { (KM18, ESN04) }\end{array}$ & $\begin{array}{l}\text { Information + Content Quality } \\
(\text { ESN } 18, \text { OSN20) }\end{array}$ & \\
\hline & $\begin{array}{l}\text { Compatibility } \\
\text { (ESN03) }\end{array}$ & $\begin{array}{l}\text { Performance Measurement } \\
\text { (KM11, KM17, ESN32, OSN07) }\end{array}$ & & \\
\hline & $\begin{array}{l}\text { Result Demonstrability } \\
\text { (ESN02) }\end{array}$ & $\begin{array}{l}\text { KM+ Human Resources } \\
(\mathrm{KM} 08, \mathrm{KM} 10, \mathrm{OSN} 08)\end{array}$ & & \\
\hline & & $\begin{array}{l}\text { Define responsibilities } \\
\text { (OSN09) }\end{array}$ & & \\
\hline & & $\begin{array}{l}\text { Team }+P M \\
(\text { OSN11) }\end{array}$ & & \\
\hline & & $\begin{array}{l}\text { Fitting Organizational Culture } \\
+ \text { Structure } \\
\text { (KM02, KM06, KM13, KM19, } \\
\text { OSN17) }\end{array}$ & & \\
\hline & & $\begin{array}{l}\text { Adequate Processes } \\
\text { (KM05) }\end{array}$ & & \\
\hline & & $\begin{array}{l}\text { Providing No Alternatives } \\
\text { (OSN03) }\end{array}$ & & \\
\hline
\end{tabular}

The starting point of the KM project was an already conducted first attempt to facilitate knowledge exchange and development based on a wiki. However, it was done by the provide-and-pray approach [7], leading to a failure in the acceptance of the system.

Besides the considerations of [27], possible reasons for the failure of this initial wiki implementation had to be analyzed in depth. In addition to this, the project team wanted to get a grounded understanding of the current sentiment toward the IT systems in use and knowledge-sharing readiness at the organization. Therefore, a nationwide online survey with 939 participants (organization's employees) was conducted to identify user perspectives and user needs for a promising collaboration tool as well as to analyze potential reasons for the failure of the organizations first approach. According to the results, $74 \%$ of the participants stated that information and files are stored in too many different places, ultimately resulting in the employees' confusion on where to find desired documents, as well as leading to the creation of redundancies organization wide. Staying with the argument about difficulties with information retrieval, $45 \%$ of the users criticized the insufficient search functions of available IT systems, which in effect leads to the loss of efficiencies as well as to the loss of quality in operative work because the desired information could not be found. Another problem, according to $39 \%$ of users, is the existence of purely word-of-mouth information. By looking at what is important to the users, it became clear that collaboration with colleagues (either verbal or via text) is an essential component of the employees' operative work. Therefore, $82.7 \%$ considered this to be essential. In addition to this, $79.7 \%$ are generally willing to share their knowledge with colleagues. However, a major motivator is the provision of adequate incentives (either monetary or nonmonetary, such as colleagues' appreciation, as stated in the survey). By looking at the usage of the initial Wiki in detail, a total of $46 \%$ claimed to not use the system at all. As a possible reason for this, several statements were given: the lack of qualitative content, the unappealing usability, and the uncertainty relating to where to search for information due to other available IT systems (redundancies). Summarizing the insights of the nationwide survey, four major fields of action for the development and implementation of a social KMS could be identified: redundancy elimination, collaboration facilitation, search functionalities enhancement and participation promotion. 
The decision to implement a new wiki as a KMS can be explained by using several arguments. First, a wiki is a well-established software tool in today's society as can be illustrated by the world's largest wiki platform, Wikipedia, which receives over 5.7 billion visits a day worldwide [36]. Furthermore, a wiki can make use of various extensions to facilitate collaboration via social media functionalities due to its open-source character.

The TOSI framework for a social KMS was referred to in order to identify the most relevant CSFs in the eyes of the organization. The identification was conducted via semi-structured interviews with the top management, where the above-mentioned major fields for improvements set the scene (redundancy elimination, collaboration facilitation, search functionalities enhancement, and participation promotion). In order to get the most complete view on all possible CSFs, all four dimensions of the TOSI framework were considered and discussed. Furthermore, to evaluate the relevance of a single $\mathrm{CSF}$, the top management allocated points $(0=$ not relevant; 3 = highly relevant) to the CSFs and at the end of the discussions and interviews, the two most relevant CSFs of each dimension were selected for the consideration as part of an adequate implementation strategy (see Table 5).

Table 5. Selected CSFs for the Wiki

\begin{tabular}{r|l}
\hline $\mathbf{T}$ & System Integration \\
\hdashline $\mathbf{O}$ & Usability \\
\hdashline $\mathbf{S}$ & Top Management Support \\
$\mathbf{S}$ & Critical Mass \\
\hdashline $\mathbf{I}$ & Tolerance of Failure \\
\hdashline $\mathbf{I}$ & Time \\
\hline
\end{tabular}

Considering the first aspect from the fields of action (redundancy elimination), the CSF system integration was seen as essential by the whole top management team. On this matter, one interviewee stated that "...even though it might not be possible to eliminate all redundancies, integrating the wiki into the operative work will contribute greatly to a major reduction of current redundancies." This can be justified with the fact that the wiki will be more visible and easier accessible for the employees and might therefore develop to be their first contact point when looking for information. The interviewees also suggested to define clear rules on what information to find where, which is intended to help reduce redundancies. Another aspect for the elimination of redundancies is the provision of suitable education and training for the new wiki system. On this matter, the employees will learn how to use the tool, which decreases entry barriers regarding the acceptance and usage of the wiki, as state by one manager.

Collaboration facilitation as a field of action is managed by two CSFs. On the one hand, the top management found it essential to focus on reaching a critical mass. They justified their decision using the common chicken-and-egg problem. Therefore, a plan was mapped out to fill the wiki with content before the roll out, which results in an additional benefit for the users since they can use the wiki for knowledge retrieval right away. This also leads to considering another CSF, advantage for work. The top management discussed this point in more detail, since some felt that collaboration could only be facilitated via incentives such as pay raises or other similar methods. However, the majority of the interviewees had a different opinion. They argued that monetary incentives might only have a short-term benefit and will not help the organization nor the individual employee in the long run. As for this matter, they agreed that sustainable collaboration could only be reached if the employees could see advantages for their work, meaning that if one employee found useful information regarding his or her problem, there is a higher chance that this employee might also contribute by writing an article in order to help others as well.

Regarding the enhancement of search functionalities, the CSF usability plays a major role. Referring to the online survey again, users often found the search results of the intranet confusing, since it did not provide them with the desired information. The initial wiki did provide good search functionalities; however, due to a non-appealing design, the system and the search function were not seen as useful. To solve this issue, a new appealing design was developed where simplicity was the main focus. Accordingly, one manager stated that many of his team members are not very IT savvy, resulting in the rejection of any non-intuitive system. As a result of good usability, the search functionalities were enhanced implicitly, which ultimately leads to increased efficiency in the operative work.

As for the last field of action, it was interesting to see that all interviewees reached a consensus right away, stating that participation promotion must be supported and empowered by the top management and team leaders. On this matter, the first identified CSF was time meaning that leaders must give their employees enough time and open space to be able to contribute (e.g., write articles for the wiki) in addition to their primary tasks. The CSF top management support was also chosen; however, due to the fact that the entire management supports $\mathrm{KM}$ and its 
developments, this CSF could already be seen as fulfilled. As a third allocated CSF supporting participation, tolerance of failure was selected. Admittedly, this CSF was discussed the most and was also allocated the least points of the selected CSFs. By taking a closer look, this result becomes evident. Being an organization of the public sector where correctness and legal certainty play an immense role, there is no room for mistakes or failures. Therefore, some interviewees disagreed with tolerance of failure being a CSF for the organization. However, the majority of the top management agreed that a certain amount of tolerance of failure is critical for the employees to be willing to share their knowledge or, more specifically, to contribute to the wiki. Otherwise, the wiki would transform into a unilateral knowledge storage system, where only official statements are stored and no best practices are available, since the risk of publishing potential false information is too high.

\section{Conclusion}

This publication identified the necessity of a holistic CSF framework for the implementation of a social KMS. On this matter, the established TOSI framework was adapted and used to identify CSFs in the areas of KM, ESN and OSN. Which were then matched to the framework and its respective dimensions in order to have a model at hand that could be consulted for implementing a social KMS. To demonstrate its applicability in practical settings, the author used the framework for the implementation of a wiki at a public sector organization.

This paper contributes to both theory and practice. As a contribution to theory, CSFs regarding a social KMS were identified, consolidated and matched to the TOSI framework. From a practical perspective, the TOSI framework provided the possibility to identify relevant CSFs for an organization by considering all dimensions to get the best fit and therefore to help with the implementation of a social KMS.

However, the paper at hand is not without limitations. Although the author relied on extensive literature reviews in the fields of KM, ESN and OSN, the possibility that some CSFs might have been left out cannot be excluded. Furthermore, the author does understand that a wiki is not a highly sophisticated software tool, such as an artificial intelligence (AI) solution or cloud technologies. However, as stated above, the no-cloud regulation for the public sector organization had to be considered, meaning that all widely established providers of AI and cloud technologies were excluded. A wiki, however, can be installed on a local server and can provide a wide range of customization options.

Additionally, further evaluations (e.g., via empirical studies on the whole organization) are necessary to validate the chosen CSFs in operations and to prove the CSF enhancements to the organization in more detail, such as their time saving potential via improved information retrieval or an increase in efficiency in the operative work. One possibility for this assessment could be making use of the KM success model of [37] for further research.

\section{References}

[1] Archer-Brown, C., and Kietzmann, J., "Strategic knowledge management and enterprise social media", Journal of knowledge management. 22(6), 2018, p. 12881309 .

[2] Chin, C.P.-Y., Evans, N., and Choo, K.-K.R., "Exploring factors influencing the use of enterprise social networks in multinational professional service firms", Journal of organizational computing and Electronic Commerce. 25(3), 2015, p. 289-315.

[3] Luen, T.W., and Al-Hawamdeh, S., "Knowledge management in the public sector: principles and practices in police work", Journal of information Science. 27(5), 2001, p. 311-318.

[4] Lehner, F., "Wissensmanagement: Grundlagen, Methoden und technische Unterstützung": Carl Hanser Verlag GmbH Co KG. 2019.

[5] Ragab, M.A., and Arisha, A., "Knowledge management and measurement: a critical review", Journal of knowledge management. 2013.

[6] Massaro, M., Dumay, J., and Garlatti, A., "Public sector knowledge management: a structured literature review", Journal of Knowledge Management. 2015.

[7] Gartner. "Gartner says the vast majority of social collaboration initiatives fail due to lack of purpose". 2013; Available http://www.gartner.com/newsroom/id/2402115.

[8] Jennex, M.E., "Knowledge management in modern organizations": Igi Global. 2006.

[9] Von Krogh, G., "How does social software change knowledge management? Toward a strategic research agenda", The Journal of Strategic Information Systems. 21(2), 2012, p. 154-164.

[10] Helms, R., Cranefield, J., and Van Reijsen, J., "Social Knowledge Management in Action": Springer. 2017.

[11] Kaplan, A.M., and Haenlein, M., "Users of the world, unite! The challenges and opportunities of Social Media", Business horizons. 53(1), 2010, p. 59-68. 
[12] Hester, A., "A comparative analysis of the usage and infusion of wiki and non-wiki-based knowledge management systems", Information Technology Management. 12(4), 2011, p. 335-355.

[13] Bullen, C.V., and Rockart, J.F., "A primer on critical success factors", 1981.

[14] Steinhuser, M., Smolnik, S., and Hoppe, U., "Towards a measurement model of corporate social software successevidences from an exploratory multiple case study", in 44th Hawaii International conference on system sciences, 2011: IEEE.

[15] Kügler, M., Smolnik, S., and Raeth, P., "Why don't you use it? Assessing the determinants of enterprise social software usage: a conceptual model integrating innovation diffusion and social capital theories", in Thirty Third International Conference on Information Systems (ICIS), 2012, Orlando, Florida.

[16] Gable, G.G., Sedera, D., and Chan, T., "Reconceptualizing information system success: The IS-impact measurement model", Journal of the association for information systems. 9(7), 2008, p. 18.

[17] Kulkarni, U.R., Ravindran, S., and Freeze, R., "A knowledge management success model: Theoretical development and empirical validation", Journal of management information systems. 23(3), 2006, p. 309-347.

[18] Kankanhalli, A., Tan, B.C., and Wei, K.-K., "Contributing knowledge to electronic knowledge repositories: an empirical investigation", MIS quarterly. 2005, p. 113-143.

[19] Wenpin Tsai, S.G., "Social Capital and Value Creation: The Role of Intrafirm Networks", The Academy ofManagement Journal. 41(4), 1998, p. 464-476.

[20] Yli-Renko, H., Autio, E., and Sapienza, H., "Social capital, knowledge acquisition, and knowledge exploitation in young technology-based firms", Strategic management journal. 22(6-7), 2001, p. 587-613.

[21] Delone, W.H., and McLean, E.R., "The DeLone and McLean model of information systems success: a ten-year update", Journal of management information systems. 19(4), 2003, p. 9-30.

[22] Bolisani, E., and Scarso, E., "Factors affecting the use of wiki to manage knowledge in a small company", Journal of Knowledge Management. 2016.

[23] Andersen, E., "Using wikis in a corporate context", Handbuch e-learning. 2005, p. 1-15.

[24] Davis, F.D., "A technology acceptance model for empirically testing new end-user information systems: Theory and results", 1985, Massachusetts Institute of Technology, 1985.
[25] Iglesias-Pradas, S., Hernández-García, Á., and Fernández-Cardador, P., "Social factors' influences on corporate wiki acceptance and use", Journal of Business Research. 68(7), 2015, p. 1481-1487.

[26] Kiniti, S., and Standing, C., "Wikis as knowledge management systems: issues and challenges", Journal of Systems Information Technology Management. 2013.

[27] Alqahtani, F.H., "The acceptance of corporate wiki use for knowledge diffusion purposes", Aslib Journal of Information Management. 2017.

[28] Henttonen, K., Kianto, A., and Ritala, P.J.J.o.K.M., "Knowledge sharing and individual work performance: an empirical study of a public sector organisation", 2016.

[29] Peffers, K., Tuunanen, T., Rothenberger, M.A., and Chatterjee, S., "A design science research methodology for information systems research", Journal of management information systems. 24(3), 2007, p. 45-77.

[30] Mathew, A.O., and Rodrigues, L.L., "Critical success factors of knowledge management: a review", World Review of Entrepreneurship, Management and Sustainable Development. 15(4), 2019, p. 409-427.

[31] Jennex, M., and Olfman, L., "Assessing knowledge management success", International Journal of Knowledge Management. 1(2), 2005, p. 33-49.

[32] Chin, C.P.-Y., Evans, N., Choo, K.-K.R., and Tan, F.B., "What Influences Employees to Use Enterprise Social Networks? A Socio-Technical Perspective", in Pacific Asia Conference on Information Systems (PACIS), 2015.

[33] Azaizah, N., Reychav, I., Raban, D.R., Simon, T., and McHaney, R.J.I.J.o.I.M., "Impact of ESN implementation on communication and knowledge-sharing in a multinational organization", 43, 2018, p. 284-294.

[34] Hammerl, T., Schwaiger, J.M., and Leist, S., "Measuring the Success of Social Media: Matching Identified Success Factors to Social Media KPIs", in Proceedings of the 52nd Hawaii International Conference on System Sciences. 2019, 2019.

[35] Mayring, P., "Qualitative content analysis: theoretical foundation, basic procedures and software solution", 2014.

[36] Statista. "Number of Wikipedia Visits from March 2019 to April 2020". 2020 13.05.2020]; Available from: https:/de.statista.com/statistik/daten/studie/1021463/umfra ge/anzahl-der-visits-pro-monat-von-wikipediaorg/.

[37] Jennex, M.E.O., Lorne, "A Model of Knowledge Management Success", International Journal of Knowledge Management. 2(3), 2006, p. 51-68. 